\title{
OPTIMAL STOPPING OF A BROWNIAN BRIDGE
}

\author{
ERIK EKSTRÖM*** AND \\ HENRIK WANNTORP, ${ }^{* * *}$ Uppsala University
}

\begin{abstract}
We study several optimal stopping problems in which the gains process is a Brownian bridge or a functional of a Brownian bridge. Our examples constitute natural finitehorizon optimal stopping problems with explicit solutions.

Keywords: Optimal stopping; Brownian bridge

2000 Mathematics Subject Classification: Primary 60G40
\end{abstract}

Secondary $60 \mathrm{H} 30$

\section{Introduction}

In this paper we consider when to stop a Brownian bridge, or a functional of a Brownian bridge, if performance is measured in the expected value sense. To describe a problem of this type, let $X$ be a Brownian bridge with $X_{0}=X_{1}=0$, and define the value

$$
V=\sup _{0 \leq \tau \leq 1} \mathrm{E} X_{\tau}
$$

Here the supremum is taken over all random times that are stopping times with respect to the filtration generated by $X$. A solution to the optimal stopping problem (1.1) consists of finding the value $V$ as well as determining an optimal stopping time $\tau^{*}$ for which the supremum is attained.

The optimal stopping problem (1.1) is a continuous version of the following classical urn problem. Suppose that an urn contains $n$ red balls and $n$ black balls which are drawn without replacement. Moreover, every red ball pays you a dollar and every black ball fines you a dollar. If you may stop the game at any time, what is your maximal expected profit and what strategy should you use? (In the guise of a card game, the same problem appears as Question 1.42 of [2].)

One potential application of problem (1.1) is in financial theory. Indeed, it has recently been reported that stock prices tend to end up at the strikes of heavily traded options written on the stocks when they mature; see [1] and the references therein. A possible explanation for this pinning phenomenon is that hedgers with a long position in vanilla options are advised by standard Black-Scholes theory to buy stocks if the price falls and to sell stocks if the price rises. Moreover, this trading is more significant at a strike and close to maturity since the option delta changes rapidly there. By supply and demand arguments, the stock price therefore pins at the strike. Problem (1.1) serves as a first approximation to the problem of when to sell a stock in the presence of stock pinning.

Received 8 October 2008; revision received 4 February 2009.

* Postal address: Department of Mathematics, Uppsala University, Box 480, SE-751 06 Uppsala, Sweden.

** Email address: ekstrom@math.uu.se

*** Email address: henrik.wanntorp@swedbank.se 
As is well known, most optimal stopping problems with a finite horizon lack explicit solutions. Nevertheless, using a representation of the Brownian bridge as a time-changed Brownian motion, Shepp [5] transformed (1.1) into the problem

$$
\sup _{\tau \geq 0} \mathrm{E}\left[\frac{W_{\tau}}{1+\tau}\right]
$$

for a Brownian motion $W$, which he solved explicitly. In the current paper we solve (1.1), and several related problems, using, in our opinion, a more direct approach. The method consists of the following three classical steps in optimal stopping theory; compare, for example, the recent monograph [4].

(i) Allow for an arbitrary starting point, thereby embedding the problem in a Markovian framework.

(ii) Formulate a free boundary problem for the value function and the optimal stopping boundary. Solve this free boundary problem to produce a candidate solution to the problem in (i).

(iii) Verify that this candidate solution is indeed the correct solution.

It seems to us that scheme (i)-(iii) is not only more direct, but also easier to extend than the method used in [5] in that it does not rely on the explicit representation of a Brownian bridge as a time-changed Brownian motion. For example, the same method also naturally applies to variants of problem (1.1) involving other underlying processes, such as Bessel bridges and diffusions of the type $\mathrm{d} X_{s}=-a X_{s} /(1-s) \mathrm{d} s+\mathrm{d} W_{s}$, as well as to optimal stopping problems with gain functions involving certain functionals, such as absolute values, powers, and integrals, of such processes.

In Section 2 we reproduce Shepp's solution to problem (1.1) following scheme (i)-(iii) above. In Section 3 we study related problems where the gain functions are certain functionals of the Brownian bridge. Some of these problems could be solved by first writing the Brownian bridge as a time-changed Brownian motion as in [5], and then appealing to explicit solutions to the transformed problems given in [3]. Rather than performing these transformations, we again show that the more direct approach, (i)-(iii), is applicable.

\section{Optimal stopping of a Brownian bridge}

Let $W=\left\{W_{t}\right\}_{t \geq 0}$ denote a standard Brownian motion.

Definition 2.1. A continuous process $\left\{X_{s}\right\}_{t \leq s \leq 1}$ satisfying

$$
\begin{gathered}
\mathrm{d} X_{s}=-\frac{X_{s}}{1-s} \mathrm{~d} s+\mathrm{d} W_{s}, \quad t \leq s<1, \\
X_{t}=x,
\end{gathered}
$$

is called a Brownian bridge starting from $x$ at time $t \geq 0$ and ending at 0 .

Remark. It is well known that the exploding drift term forces a Brownian bridge to satisfy $X_{1}=0$ almost surely. In fact, the Brownian bridge can alternatively be defined as a standard Brownian motion conditioned upon being 0 at time 1 . 
In this section we solve the optimal stopping problem (1.1) by following steps (i)-(iii) described in the introduction. Thus, we first embed it in a more general setting where the Brownian bridge is first observed at a time $t \in[0,1)$ at a point $x \in \mathbb{R}$. Define

$$
V(t, x)=\sup _{t \leq \tau \leq 1} \mathrm{E}_{t, x} X_{\tau}
$$

At times when $X$ is negative, the drift is positive, so it is advantageous to continue observing. On the other hand, if $X$ is positive then the drift is negative, which makes stopping attractive, and this is accentuated if $X$ is large. Indeed, the larger the current value, the more the drift term will push the process down. These considerations make it plausible that there exists a boundary $x=b(t)$ such that stopping is optimal above this boundary and continuation is optimal below this boundary. From general optimal stopping theory (see, for example, [4]), we expect the value function and the optimal stopping boundary to solve the free boundary problem

$$
\begin{gathered}
V_{t}(t, x)-\frac{x}{1-t} V_{x}(t, x)+\frac{1}{2} V_{x x}(t, x)=0 \quad \text { if } x<b(t), \\
V(t, x)=x \quad \text { if } x=b(t), \\
V_{x}(t, x)=1 \quad \text { if } x=b(t), \\
V(t, x)=0 \quad \text { if } x=-\infty
\end{gathered}
$$

for $t<1$ and $V(1,0)=0$. Here (2.2c) is the so-called smooth fit condition and (2.2d) is to be interpreted as $\lim _{x \rightarrow-\infty} V(t, x)=0$. Moreover, the first passage time,

$$
\tau^{*}=\inf \left\{s \geq t: X_{s} \geq b(s)\right\},
$$

of $X$ over the boundary $b$ should be an optimal stopping time in (2.1).

Now, the ansatz $b(t)=B \sqrt{1-t}$ and

$$
V(t, x)=\sqrt{1-t} f\left(\frac{x}{\sqrt{1-t}}\right)
$$

transforms the free boundary problem $(2.2 \mathrm{a})-(2.2 \mathrm{~d})$ into

$$
\begin{gathered}
f^{\prime \prime}(y)-y f^{\prime}(y)-f(y)=0, \quad y<B, \\
f(y)=y, \quad y=B, \\
f^{\prime}(y)=1, \quad y=B, \\
f(y)=0, \quad y=-\infty,
\end{gathered}
$$

where $y=x / \sqrt{1-t}$. The general solution to the ordinary differential equation (ODE) in (2.4a) is given by

$$
f(y)=C \Phi(y) \exp \left(\frac{y^{2}}{2}\right)+D \exp \left(\frac{y^{2}}{2}\right),
$$

where

$$
\Phi(y)=\frac{1}{\sqrt{2 \pi}} \int_{-\infty}^{y} \exp \left(\frac{-z^{2}}{2}\right) \mathrm{d} z
$$


is the distribution function of a standard normal random variable. Boundary condition (2.4d) implies that $D=0$. It is therefore straightforward to check that the unique solution to (2.4a)$(2.4 \mathrm{~d})$ is given by $(f, B)$, where

$$
f(y)= \begin{cases}\sqrt{2 \pi}\left(1-B^{2}\right) \exp \left(\frac{y^{2}}{2}\right) \Phi(y), & y<B, \\ y, & y=B,\end{cases}
$$

and $B$ is the unique solution to

$$
\sqrt{2 \pi}\left(1-B^{2}\right) \exp \left(\frac{B^{2}}{2}\right) \Phi(B)=B
$$

Remark. To see that (2.5) admits a unique solution, note that the function

$$
h(x)=\sqrt{2 \pi}\left(1-x^{2}\right) \exp \left(\frac{x^{2}}{2}\right) \Phi(x)-x
$$

is strictly decreasing for $x \geq 0$, and satisfies

$$
h(x)>0 \quad \text { for } x \leq 0 \text { and } \quad \lim _{x \rightarrow \infty} h(x)=-\infty .
$$

Remark. The ansatz (2.3) reduces the free boundary problem (2.2a)-(2.2d), which involves a partial differential equation, to the free boundary problem (2.4a)-(2.4d), which involves an ordinary differential equation. An alternative way of achieving this reduction would be to introduce the time-changed process

$$
Z_{s}=\frac{X_{\sigma_{s}}}{\sqrt{1-\sigma_{s}}}
$$

where $\sigma_{s}=1-(1-t) \mathrm{e}^{-2 s}$. This reduces problem (2.1) to a perpetual optimal stopping problem in terms of the time-homogeneous Ornstein-Uhlenbeck process $Z$; compare [3].

Via (2.3) we arrive at a candidate value function $V^{*}:[0,1) \times \mathbb{R} \cup\{(1,0)\} \rightarrow \mathbb{R}$ given by

$$
V^{*}(t, x)= \begin{cases}\sqrt{2 \pi(1-t)}\left(1-B^{2}\right) \exp \left(\frac{x^{2}}{2(1-t)}\right) \Phi\left(\frac{x}{\sqrt{1-t}}\right) & \text { if } x<b(t), \\ x & \text { if } x \geq b(t)\end{cases}
$$

for $t<1$ and $V(1,0)=0$, where

$$
b(t)=B \sqrt{1-t} .
$$

It remains to show that this candidate value function $V^{*}$ is indeed the correct solution to problem (2.1). We do this below using a standard verification argument. As mentioned in the introduction, Theroem 2.1, below, was also obtained in [5]. For an illustration of the results in Theorem 2.1, see Figure 1.

Theorem 2.1. The value function $V(t, x)$ defined in (2.1) coincides with the function $V^{*}(t, x)$ given by (2.6). Moreover, the stopping time

$$
\tau^{*}=\inf \left\{s \geq t: X_{s} \geq B \sqrt{1-s}\right\},
$$

where $B$ is the unique solution to (2.5), is optimal. 


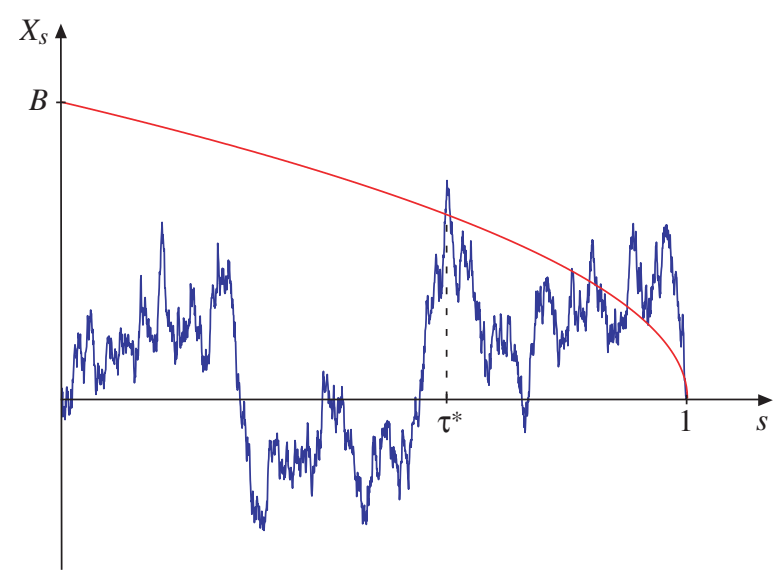

FIGURE 1: A realization of a Brownian bridge together with the optimal stopping boundary. Numerical calculations show that $B \approx 0.8399$ and $V(0,0)=\sqrt{\pi / 2}\left(1-B^{2}\right) \approx 0.3691$.

Proof. First note that $V^{*}$ satisfies

$$
V_{t}^{*}-\frac{x}{1-t} V_{x}^{*}+\frac{1}{2} V_{x x}^{*}=0 \text { for } x<b(t) .
$$

Moreover, $V_{t}^{*}$ and $V_{x}^{*}$ are continuous over $\{(t, x): x=b(t)\}$. Define the process $Y_{s}=$ $V^{*}\left(s, X_{s}\right)$, where $X$ is a Brownian bridge starting from $x$ at time $t$. An application of Itô's formula yields

$$
\begin{aligned}
\mathrm{d} Y_{s}= & \left(V_{t}^{*}\left(s, X_{s}\right)-\frac{X_{s}}{1-s} V_{x}^{*}\left(s, X_{s}\right)+\frac{1}{2} V_{x x}^{*}\left(s, X_{s}\right)\right) \mathbf{1}\left(X_{s} \neq b(s)\right) \mathrm{d} s \\
& +V_{x}^{*}\left(s, X_{s}\right) \mathrm{d} W_{s} \\
= & -\frac{X_{s}}{1-s} \mathbf{1}\left(X_{s}>b(s)\right) \mathrm{d} s+V_{x}^{*}\left(s, X_{s}\right) \mathrm{d} W_{s} \\
= & \mathrm{d} \Lambda_{s}+\mathrm{d} M_{s},
\end{aligned}
$$

where

$$
\Lambda_{s}=-\int_{t}^{s} \frac{X_{u}}{1-u} \mathbf{1}\left(X_{u}>b(u)\right) \mathrm{d} u
$$

is a decreasing process and

$$
M_{s}=\int_{t}^{s} V_{x}^{*}\left(u, X_{u}\right) \mathbf{1}\left(X_{u} \neq b(u)\right) \mathrm{d} W_{u}
$$

is a local martingale. In fact, since $V_{x}^{*}$ is bounded, the process $M$ is a martingale. Let $\tau$ be a stopping time satisfying $t \leq \tau \leq 1$. Since $V^{*}(t, x) \geq x$, we have

$$
\mathrm{E}_{t, x} X_{\tau} \leq \mathrm{E}_{t, x} V^{*}\left(\tau, X_{\tau}\right)=V^{*}(t, x)+\mathrm{E}_{t, x}\left[\Lambda_{\tau}+M_{\tau}\right] \leq V^{*}(t, x),
$$

where the last inequality follows from the optional sampling theorem. Consequently,

$$
V(t, x) \leq V^{*}(t, x)
$$


To establish the reverse inequality, note that, since $V^{*}\left(\tau^{*}, X_{\tau^{*}}\right)=X_{\tau^{*}}$ and $\Lambda_{\tau^{*}}=0$, both inequalities in (2.7) reduce to equalities for $\tau=\tau^{*}$. Thus,

$$
V(t, x) \geq \mathrm{E}_{t, x} X_{\tau^{*}}=V^{*}(t, x),
$$

which finishes the proof.

Remark. The pinning time 1 and the pinning level 0 can of course be easily generalised. If the process $X$ is defined by

$$
\mathrm{d} X_{s}=-\frac{X_{s}-a}{T-s} \mathrm{~d} s+\mathrm{d} W_{s},
$$

then $X$ pins at $a$ at time $T$. The value function $V(t, x)=\sup _{t \leq \tau \leq T} \mathrm{E} X_{\tau}$ is then given by

$$
V(t, x)= \begin{cases}a+\sqrt{2 \pi(T-t)}\left(1-B^{2}\right) \exp \left(\frac{(x-a)^{2}}{2(T-t)}\right) \Phi\left(\frac{x-a}{\sqrt{T-t}}\right) & \text { if } x<b(t), \\ x & \text { if } x \geq b(t)\end{cases}
$$

where $b(t)=a+B \sqrt{T-t}$ and $B$ is as in Theorem 2.1. Moreover,

$$
\tau^{*}=\inf \left\{s \geq t: X_{s} \geq a+B \sqrt{T-s}\right\}
$$

is an optimal stopping time.

\section{Optimal stopping of a functional of a Brownian bridge}

In this section we consider different variants of problem (1.1), all of which admit explicit solutions. We refrain from performing the rigorous verification arguments, since they all follow along the same lines as in the proof of Theorem 2.1. Instead, we give the intuition behind finding a candidate solution.

\subsection{Odd powers of a Brownian bridge}

Let $X$ be a Brownian bridge and consider the optimal stopping problem

$$
V(t, x)=\sup _{t \leq \tau \leq 1} \mathrm{E}_{t, x} X_{\tau}^{2 n+1}
$$

where $n \geq 1$ is an integer. The value function and the optimal stopping boundary should solve the free boundary problem

$$
\begin{gathered}
V_{t}(t, x)-\frac{x}{1-t} V_{x}(t, x)+\frac{1}{2} V_{x x}(t, x)=0 \quad \text { if } x<b(t), \\
V(t, x)=x^{2 n+1} \quad \text { if } x=b(t), \\
V_{x}(t, x)=(2 n+1) x^{2 n} \quad \text { if } x=b(t), \\
V(t, x)=0 \quad \text { if } x=-\infty,
\end{gathered}
$$

for $t<1$ and $V(1,0)=0$. The ansatz $b(t)=B \sqrt{1-t}$ and

$$
V(t, x)=(1-t)^{n+1 / 2} f\left(\frac{x}{\sqrt{1-t}}\right)
$$


transforms the free boundary problem $(3.2 \mathrm{a})-(3.2 \mathrm{~d})$ into

$$
\begin{gathered}
f^{\prime \prime}(y)-y f^{\prime}(y)-(2 n+1) f(y)=0 \quad \text { if } y<B, \\
f(y)=y^{2 n+1} \quad \text { if } y=B, \\
f^{\prime}(y)=(2 n+1) y^{2 n} \quad \text { if } y=B, \\
f(y)=0 \quad \text { if } y=-\infty .
\end{gathered}
$$

The general solution to the ODE in (3.4a) is given by

$$
f(y)=C G_{2 n+1}(y)+D F_{2 n+1}(y),
$$

where

$$
G_{v}(y)=\int_{0}^{\infty} u^{\nu-1} \exp \left(\frac{-y u-u^{2}}{2}\right) \mathrm{d} u \quad \text { and } \quad F_{v}(y)=G_{v}(-y) .
$$

Using the boundary conditions, we find that $C=0$ and that the unique solution to (3.4a)-(3.4d) is given by $(f, B)$, where

$$
f(y)=B^{2 n+1} \frac{F_{2 n+1}(y)}{F_{2 n+1}(B)}, \quad y<B,
$$

and $B$ satisfies

$$
B F_{2 n+1}^{\prime}(B)=(2 n+1) F_{2 n+1}(B) .
$$

Via (3.3) we obtain the following result.

Theorem 3.1. The value function $V$ defined in (3.1) is given by

$$
V(t, x)= \begin{cases}(1-t)^{n+1 / 2} B^{2 n+1} \frac{F_{2 n+1}(x / \sqrt{1-t})}{F_{2 n+1}(B)}, & x<B \sqrt{1-t}, \\ x^{2 n+1}, & x \geq B \sqrt{1-t},\end{cases}
$$

where $B$ is the unique solution to (3.6). Moreover, the stopping time

$$
\tau^{*}=\inf \left\{s \geq t: X_{s} \geq B \sqrt{1-s}\right\}
$$

is optimal.

We again emphasize that the above derivation is merely heuristic, and that a formal proof requires a verification argument similar to the one performed in the previous section.

Remark. In order to verify that (3.6) indeed has a unique solution, we note that the function

$$
h(x)=(2 n+1) F_{2 n+1}(x)-x F_{2 n+1}^{\prime}(x)
$$

satisfies $h(x)>0$ for $x \leq 0$ and that $h(\sqrt{2 n+1}) \leq 0$. It thus suffices to show that the quotient

$$
g(x)=\frac{x F_{2 n+1}^{\prime}(x)}{F_{2 n+1}(x)}
$$

is strictly monotone for $x>0$. Differentiating $g$ we find that $g^{\prime}(x)>0$, provided that $\mathrm{E} U^{2}>(\mathrm{E} U)^{2}$, where $U$ is a random variable with density

$$
f_{U}(u)=\frac{u^{2 n} \exp \left(x u-u^{2} / 2\right)}{\int_{0}^{\infty} u^{2 n} \exp \left(x u-u^{2} / 2\right) \mathrm{d} u} \quad \text { for } u>0 .
$$

The claimed uniqueness thus follows from (a strict version of) Jensen's inequality. 


\subsection{Reflected Brownian bridge}

Let $X$ be a Brownian bridge, and consider the optimal stopping problem

$$
V(t, x)=\sup _{t \leq \tau \leq 1} \mathrm{E}_{t, x}\left|X_{\tau}\right|^{q},
$$

where $q>0$.

Theorem 3.2. The value function $V$ in (3.7) satisfies

$$
V(t, x)= \begin{cases}(1-t)^{q / 2} B^{q} \frac{G_{q}(x / \sqrt{1-t})+F_{q}(x / \sqrt{1-t})}{G_{q}(B)+F_{q}(B)}, & |x|<B \sqrt{1-t}, \\ |x|^{q}, & |x| \geq B \sqrt{1-t},\end{cases}
$$

where the functions $G$ and $F$ are given by (3.5), and $B$ is the unique positive solution to

$$
B\left(G_{q}^{\prime}(B)+F_{q}^{\prime}(B)\right)=q\left(G_{q}(B)+F_{q}(B)\right) .
$$

Moreover, the stopping time

$$
\tau^{*}=\inf \left\{s \geq t:\left|X_{s}\right| \geq B \sqrt{1-s}\right\}
$$

is optimal in (3.7).

The intuition is as follows. We expect the continuation region to be of the form

$$
\{(t, x):-b(t)<x<b(t)\}
$$

for some function $b$, and the value function and the optimal stopping boundary should solve the free boundary problem

$$
\begin{gathered}
V_{t}(t, x)-\frac{x}{1-t} V_{x}(t, x)+\frac{1}{2} V_{x x}(t, x)=0 \quad \text { if }|x|<b(t), \\
V(t, x)=|x|^{q} \quad \text { if }|x|=b(t), \\
V_{x}(t, x)=\operatorname{sgn}(x) q|x|^{q-1} \quad \text { if }|x|=b(t),
\end{gathered}
$$

for $t<1$ and $V(1,0)=0$. Moreover, due to the symmetry in the problem, the solution should be an even function in $x$. The ansatz $b(t)=B \sqrt{1-t}$ and

$$
V(t, x)=(1-t)^{q / 2} f\left(\frac{x}{\sqrt{1-t}}\right)
$$

transforms the free boundary problem into

$$
\begin{gathered}
f^{\prime \prime}(y)-y f^{\prime}(y)-q f(y)=0 \quad \text { if }|y|<B, \\
f(y)=|y|^{q} \quad \text { if }|y|=B, \\
f^{\prime}(y)=\operatorname{sgn}(y) q|y|^{q-1} \quad \text { if }|y|=B .
\end{gathered}
$$

The general solution to the ODE in (3.10a) is given by

$$
f(y)=C G_{q}(y)+D F_{q}(y) .
$$


Since $f$ should be even, we conclude that $C=D$, and using the boundary conditions, we find that the unique solution to (3.10a)-(3.10c) is given by $(f, B)$, where

$$
f(y)=B^{q} \frac{G_{q}(y)+F_{q}(y)}{G_{q}(B)+F_{q}(B)}, \quad|y|<B,
$$

and $B$ satisfies (3.8). Using (3.9), we arrive at the (candidate) value function given in Theorem 3.2.

Remark. The uniqueness of the solution in (3.8) can be verified by proceeding as in the case of odd powers of a Brownian bridge.

Remark. For some values of $q$, the solution admits a more explicit form. For example, in the case in which $q=2 n+1$, where $n$ is a nonnegative integer, we have

$$
G_{2 n+1}(y)+F_{2 n+1}(y)=C_{n} \frac{\mathrm{d}^{2 n}}{\mathrm{~d} y^{2 n}} \exp \left(\frac{y^{2}}{2}\right)
$$

for some constant $C_{n}$. The problem $q=1$ was studied in [5], and its solution is remarkably simple. Indeed, in that case, $B=1$ and the value function is given by

$$
V(t, x)= \begin{cases}\sqrt{1-t} \exp \left(\frac{x^{2}}{2(1-t)}-\frac{1}{2}\right), & |x|<\sqrt{1-t} \\ |x|, & |x| \geq \sqrt{1-t}\end{cases}
$$

\subsection{The integral of a Brownian bridge}

Consider the optimal stopping of the integral of a Brownian bridge, i.e.

$$
V(t, x)=\sup _{t \leq \tau \leq 1} \mathrm{E}_{t, x} \int_{t}^{\tau} X_{u} \mathrm{~d} u .
$$

Theorem 3.3. The value function $V$ is given by

$$
V(t, x)= \begin{cases}\frac{1}{2}(1-t) x+(1-t)^{3 / 2} \frac{B}{2} \frac{G_{3}(x / \sqrt{1-t})}{G_{3}(-B)}, & x>-B \sqrt{1-t}, \\ 0, & x \leq-B \sqrt{1-t}\end{cases}
$$

where $B$ is the unique solution to

$$
B G_{3}^{\prime}(-B)+G_{3}(-B)=0
$$

Moreover, the stopping time

$$
\tau^{*}=\inf \left\{s \geq t: X_{s} \leq-B \sqrt{1-s}\right\}
$$

is optimal. 
Again, we provide the heuristics behind the proof, but omit the formal verification. Note that we expect the value function and the optimal stopping boundary to solve the free boundary problem

$$
\begin{gathered}
V_{t}(t, x)-\frac{x}{1-t} V_{x}(t, x)+\frac{1}{2} V_{x x}(t, x)=-x \quad \text { if } x>b(t), \\
V(t, x)=0 \quad \text { if } x=b(t), \\
V_{x}(t, x)=0 \quad \text { if } x=b(t),
\end{gathered}
$$

for $t<1$ and $V(1,0)=0$. With $b(t)=-B \sqrt{1-t}$ and

$$
V(t, x)=(1-t)^{3 / 2} f\left(\frac{x}{\sqrt{1-t}}\right),
$$

the free boundary problem transforms into

$$
\begin{gathered}
f^{\prime \prime}(y)-y f^{\prime}(y)-3 f(y)=-2 y \quad \text { if } y>-B, \\
f(y)=0 \quad \text { if } y=-B, \\
f^{\prime}(y)=0 \text { if } y=-B .
\end{gathered}
$$

The general solution to this ODE is given by

$$
f(y)=C G_{3}(y)+D F_{3}(y)+\frac{1}{2} y .
$$

The solution should be of linear growth as $y$ tends to $\infty$, so therefore $D=0$. It is then straightforward to check that the unique solution to (3.13a)-(3.13c) of linear growth is given by $(f, B)$, where

$$
f(y)= \begin{cases}\frac{B}{2} \frac{G_{3}(y)}{G_{3}(-B)}+\frac{1}{2} y & \text { if } y>-B, \\ 0 & \text { if } y=-B,\end{cases}
$$

and $B$ satisfies (3.11). From (3.12) we obtain the candidate value function specified in Theorem 3.3.

Remark. To see that (3.11) admits a unique solution, note that the function

$$
h(x)=x G_{3}^{\prime}(-x)+G_{3}(-x)
$$

is decreasing for $x \geq 0$ and satisfies $h(1)<0$. Moreover, $h(x)>0$ for $x \leq 0$.

Remark. The more general problem

$$
V(t, x)=\sup _{t \leq \tau \leq 1} \mathrm{E}_{t, x} \int_{t}^{\tau} X_{u}^{2 n+1} \mathrm{~d} u,
$$

where $n \geq 0$ is an integer, can be treated as above. Indeed, the ansatz

$$
V(t, x)=(1-t)^{n+3 / 2} f\left(\frac{x}{\sqrt{1-t}}\right)
$$

transforms the corresponding free boundary problem into a time-independent one. We omit the details of this problem. 


\section{References}

[1] Avellaneda, M. and Lipkin, M. D. (2003). A market-induced mechanism for stock pinning. Quant. Finance 3, 417-425.

[2] Crack, T. F. (2007). Heard on the Street: Quantitative Questions from Wall Street Job Interviews. 10th edn.

[3] Pedersen, J. L. ANd Peskir, G. (2000). Solving non-linear optimal stopping problems by the method of timechange. Stoch. Anal. Appl. 18, 811-835.

[4] Peskir, G. And Shiryaev, A. (2006). Optimal Stopping and Free-Boundary Problems (Lectures Math. ETH Zürich). Birkhäuser, Basel.

[5] Shepr, L. A. (1969). Explicit solutions to some problems of optimal stopping. Ann. Math. Statist. 40, 993-1010. 